\title{
Comparative Semantics for a Real-Time Programming Language with Integration ${ }^{1}$
}

\author{
Franck van Breugel ${ }^{2}$ \\ Department of Mathematics and Computer Science, Vrije Universiteit \\ De Boelelaan 1081a, 1081 HV Amsterdam
}

\begin{abstract}
An operational and a denotational semantic model are presented for a real-time programming language incorporating the concept of integration. Integration enables us to specify a restricted form of unbounded non-determinism. For example, the execution of an action at an arbitrary moment in a time interval can be specified using the concept of integration. The operational and the denotational model are proved to be equivalent using a general method based on higher-order transformations and complete metric spaces. Banach's fixed point theorem, which states that a contraction on a complete metric space has a unique fixed point, will be used to define and to compare semantic models. Also Michael's theorem, which roughly states that a compact union of compact sets is compact, will be used for the definition of semantic models.
\end{abstract}

\section{Introduction}

Real-time programming can be viewed as traditional concurrency supplied with timing constraints [31]. Because these timing constraints cause more complexity, the advantages of high-level languages are even greater in real-time programming than in concurrency and sequential programming. Several languages, like RTL [22], have been designed specifically for real-time programming. Other languages are extensions of already existing languages, for example, the language TCSP [14] is an extension of the language CSP [21]. In real-time programming the correctness of a program depends not only on the flow of control. The program should also meet its timing constraints [30]. Therefore new semantic models should be developed. Several models both operational $[2,16,19]$ and denotational $[15,24,27]$ have already been provided.

In this paper a simple real-time programming language is studied. Apart from the traditional programming constructs, this language incorporates timed atomic actions and integration. Timed atomic actions are atomic actions each provided with some timing information. This timing information denotes when the atomic action should be executed. The concept of integration has been introduced in [2]. A statement is integrated over a time set, which is a subset of the time domain, i.e. a non-deterministically chosen value from the time set is passed to the statement. Integration enables us to specify the execution of an action at an arbitrary moment in a time interval, for example, $\int_{t \in[1.03,2.41]}(a, t)$ denotes the execution of the action $a$ at an arbitrary moment in the time interval $[1.03,2.41]$. This execution can give rise to an infinite (conceptually uncountable) number of different executions.

\footnotetext{
${ }^{1}$ extended abstract of [12]

2 electronic mail address: franck@cs.vu.nl
} 
For this simple real-time programming language an operational and a denotational semantic model are presented. The operational model is based on a labelled transition system [18]. The denotational model is by definition compositional and fixed points are exploited to handle recursion. This denotational model uses a complete metric space as its mathematical domain $[9,25]$.

To compare these models a method based on higher-order transformations is used [23]. This general method for comparing different semantic models has already been applied successfully to several programming paradigms varying from notions related to concurrency [7] to notions related to logic programming [4] and object-oriented programming [28]. The present paper shows another application of this technique. This method is founded on complete metric spaces. Higher-order transformations are used to define semantic operators and both operational and denotational models. Furthermore, the higher-order transformations are also used to compare operational and denotational models.

In the definition of semantic models and in the comparison of these models we use several aspects of complete metric spaces. Banach's theorem states that if $X$ is a complete metric space and $f: X \rightarrow X$ is a contraction then $f$ has a unique fixed point. This theorem will be used to define semantic models and to compare these models. Furthermore, in combination with fixed point induction Banach's fixed point theorem will be used to prove several properties of these semantic models. Michael's theorem states that if $\mathcal{X}$ is a compact set of compact sets then the set $\bigcup \mathcal{X}$ is also compact. This theorem will be used to define semantic models. Kuratowski's theorem states that if $X$ is a complete metric space then $\mathcal{P}_{n c}(X)$, the set of non-empty compact subsets of $X$, provided with the Hausdorff metric based on the metric of $X$ is again a complete metric space. We will use this theorem to obtain a complete metric space as the mathematical domain of the semantic models.

In the first section of this paper, the language definition of the real-time programming language studied in this paper is given. The second section contains some mathematical preliminaries considering metric spaces. An operational and denotational semantic model are presented in section 3 and 4 respectively. These semantic models are compared in section 5 .

In this paper a denotational modelling of integration is presented. This concept of integration describes a restricted form of unbounded non-determinism. In general, the modelling of unbounded non-determinism causes serious technical problems $[1,20]$. Because higher-order transformations are used, infinite computations can be modelled. This combination of metrically modelling a restricted form of unbounded non-determinism and infinite computations has not been presented elsewhere $[11,26]$. Because the denotational model is compared with an operational model, which captures the computational intuition, we can derive the correctness of this denotational model with respect to the operational model. Banach's fixed point theorem and Michael's theorem play a technical but eminent role as will become clear in the rest of this paper.

\section{Language definition}

In this section we introduce the syntax of the real-time programming language, which is studied in this paper. This programming language is an extension of one of the languages studied in [7]. The language is uniform, i.e. the elementary actions are left atomic [5]. The 
language is built from atomic actions provided with some timing information, sequential composition, non-deterministic choice, parallel composition, so-called integration and recursion.

The real-time concepts of this language are timed atomic actions and integration. With timed atomic actions we denote atomic actions each provided with an expression. The evaluation of this expression yields an element of the time domain denoting the amount of time the atomic action should be executed after its enabling. The concept of integration has been introduced in [2]. Integration of a statement over some time set, which is a subset of the time domain, gives rise to the execution of the statement with some non-deterministically chosen value from the time set passed to that statement.

Before we can introduce the syntax of expressions, which are part of timed atomic actions, we first introduce the following sets:

- the set $\mathbb{R}_{>}$of positive real numbers, with typical element $r$, which is our time domain;

- the set TVar of time variables, with typical element $t$;

- the set FSym of function symbols, with typical element $f$.

With each function symbol $f$ we associate a function $f: \mathbb{R}_{>}^{n} \rightarrow \mathbb{R}_{>}$. We have to restrict the functions to continuous functions in order to be able to model integration as will become clear in section 4 .

Definition 1.1 The class Exp of expressions, with typical element $e$, is given by $e::=t \mid f\left(e_{1}, \ldots, e_{n}\right)$

Because expressions are built from function symbols and time variables, expressions thernselves can also be associated with continuous functions. We will denote the value of an expression $e$ by $\mathcal{V}(e)$. After having defined the syntax of expressions, we have to introduce the following sets in order to be able to define the class of statements:

- the (possibly infinite) set Atom of atomic actions, with typical element $a$;

- the set $P V a r$ of procedure variables, with typical element $x$;

- the collection of time sets, which is represented by the set $\mathcal{P}_{n c}\left(I_{>}\right)$of non-empty compact subsets of $\mathbb{R}_{>}$, with typical element $T$.

Integration gives rise to a non-deterministic choice of an element from a time set. To guarantee that we can always make such a choice we have to restrict time sets to nonempty subsets of $\boldsymbol{R}_{>}$. The restriction of time sets to compact subsets of $\boldsymbol{I R}>$ has a technical motivation and this restriction will be crucial for the modelling of integration.

Definition 1.2 The class Stat of statements, with typical element $s$, is given by $s::=(a, e)|x| s_{1} ; s_{2}\left|s_{1} \cup s_{2}\right| s_{1} \| s_{2} \mid \int_{t \in T} s$

A statement $s$ is of one of the six following forms:

- $(a, e)$ : a timed atomic action: the atomic action $a$ has to be executed at time $\mathcal{V}(e)$, the value of the expression $e$, after its enabling; 
- $x$ : a procedure call: execution of the corresponding body of the procedure $x$;

- $s_{1} ; s_{2}$ : sequential composition of the statements $s_{1}$ and $s_{2}$;

- $s_{1} \cup s_{2}$ : non-deterministic choice of the statements $s_{1}$ and $s_{2}$;

- $s_{1} \| s_{2}$ : parallel composition of the statements $s_{1}$ and $s_{2}$ : the arbitrary interleaving of the atomic actions of both statements;

- $\int_{t \in T} s:$ integration: execution of the statement $s$ with an arbitrary element of $T$ passed to the time variable $t$ in $s$.

The execution of the statement $(a, 1.56)$ corresponds to the execution of the atomic action $a$. The atomic action $a$ should be executed 1.56 after its enabling. The execution of the integration $\int_{t \in[0.82,1.73]}(a, f(t))$ corresponds to the execution of the statement $(a, f(t))$ with a non-deterministically chosen value from the time set $[0.82,1.73]$ passed to the time variable $t$ in that statement, which can give rise to the execution of, for example, $(a, f(1.08))$. Because the evaluation of an expression delivers a positive real number, two successive atomic actions cannot be executed at the same time. We stipulate that the execution of atomic actions and operators takes no time. We refer to [10] for a justification of this assumption. Next we introduce the class of guarded statements, which will be used to define procedure bodies.

Definition 1.3 The class GStat of guarded statements, with typical element $g$, is given by

$$
g::=(a, e)|g ; s| g_{1} \cup g_{2}\left|g_{1} \| g_{2}\right| \int_{t \in T} g
$$

Before we give the definition of the class of declarations, which bind procedure variables with their corresponding bodies, we introduce the notion of free time variables. The mapping tvar gives for each statement the set of free time variables occurring in that statement. A statement is called closed whenever it does not contain any free time variables. In several cases we will restrict ourselves to the class CStat of closed statements or the class CGStat of closed guarded statements.

Definition 1.4 The class Decl of declarations, with typical element $d$, consists of sets of pairs $\left\{\left(x_{i}, g_{i}\right) \in P \operatorname{Var} \times\right.$ CGStat $\left.\mid 1 \leq i \leq n\right\}$, where $x_{i}$ are distinct procedure variables.

All procedure bodies in a declaration are restricted to guarded statements. This requirement corresponds to the usual Greibach condition in formal language theory. There are possibilities to eliminate this restriction as is illustrated in [26, 27]. However, by eliminating this restriction we are not able to model recursion any more. The restriction of the procedure bodies to closed statements guarantees that there are no global time variables. The execution of the procedure call $x$, where $x$ is declared as $(a, 1) ; x$, corresponds to the execution of the procedure body $(a, 1) ; x$. We can conclude this section with the definition of the class of programs.

Definition 1.5 The class Prog of programs, with typical element $p$, consists of pairs $(d, s)$, such that each procedure variable occurring in $s$ or $d$ is declared in $d$ and $s \in$ CStat. 


\section{Metric spaces}

Before the operational and denotational model are presented, we pay some attention to metric spaces. We will introduce a number of complete metric spaces, which will be used in the rest of this paper. For further reference considering metric spaces we suggest [8].

First of all, we define the so-called discrete metric on the class of atomic actions and on the class of statements. Furthermore, we extend our time domain $I R_{>}$to the set $\mathbb{I}_{\geq}$of non-negative real numbers in order to obtain a complete metric space. As will become clear in section 4 each mapping $d_{\mathbb{R}_{\geq}}: \mathbb{R}_{\geq} \times \mathbb{R}_{\geq} \rightarrow[0,1]$ defining a complete metric space suffices. The metric $d_{\mathbb{R} \geq}$ specifies the collection of compact subsets of the time domain: the collection of time sets. We can define a metric on $\mathbb{R}_{\geq}$as follows.

Definition 2.1 The mapping $d_{\mathbb{Z} R_{\geq}}: \mathbb{R}_{\geq} \times \mathbb{R _ { \geq }} \rightarrow[0,1]$ is given by

$$
d_{\mathbb{R}_{\geq}}\left(r, r^{\prime}\right)=\frac{\left|r-r^{\prime}\right|}{\left|r-r^{\prime}\right|+1}
$$

With respect to this metric closed intervals are compact sets. Furthermore, this metric and the usual metric on $\mathbb{Z R _ { \geq }}, d_{\mathbb{R}_{\geq}}\left(r, r^{\prime}\right)=\left|r-r^{\prime}\right|$, are equivalent, i.e. both induce the same converging behaviour. However, the metric of definition 2.1 is restricted to $[0,1]$. Next we introduce the class of timed actions. These timed actions will be used to describe the execution of timed atomic actions.

Definition 2.2 The class $T A$ of timed actions, with typical element $\alpha$, is given by $T A=A$ tom $\times \mathbb{I R}_{\geq}$

With the timed action $(a, 2)$ we will describe the execution of the atomic action $a$. The atomic action $a$ is executed 2 after its enabling. To describe the execution of a sequence of timed atomic actions we introduce timed streams.

Definition 2.3 The class TS of timed streams, with typical element $\sigma$, is given by the domain equation

$T S \cong T A \bar{\cup} T A \times i d_{\frac{1}{2}}(T S)$

For example, the timed stream $\langle(a, 2),\langle(b, 1),(c, 3)\rangle\rangle$ describes the execution of atomic action $a$ at 2 (after its enabling) followed by the execution of atomic action $b$ at 1 and the execution of atomic action $c$ at 3 . Furthermore, we have that, for example,

$$
\begin{aligned}
d_{T S}(< & (a, 2),(b, 1)>,<(a, 3),(c, 1)>) \\
& =\max \left\{d_{T A}((a, 2),(a, 3)), \frac{1}{2} d_{T S}((b, 1),(c, 1))\right\} \\
& =\max \left\{d_{T A}((a, 2),(a, 3)), \frac{1}{2} d_{T A}((b, 1),(c, 1))\right\} \\
& =\max \left\{\max \left\{d_{A t o m}(a, a), d_{\mathbb{R}_{\geq}}(2,3)\right\}, \frac{1}{2} \max \left\{d_{\text {Atam } \left.\left.(b, c), d_{\mathbb{R}_{\geq}}(1,1)\right\}\right\}}\right.\right. \\
& =\max \left\{\max \left\{0, \frac{1}{2}\right\}, \frac{1}{2} \max \{1,0\}\right\} \\
& =\frac{1}{2}
\end{aligned}
$$

Sets of sequences of timed atomic actions will be described by non-empty compact sets of timed streams. Finally, we introduce the class of substitutions and define a metric on this class.

Definition 2.4 The class $S u b s t$ of substitutions, with typical element $\theta$, consists of the class of homomorphisms from $E x p$ to $\mathbb{I R}_{\geq}$. 
We will only consider substitutions $\theta$ with a finite support, i.e. there exist only finitely many time variables $t$ such that $\theta t \neq t$. To simplify the exposition we will, without loss of generality, assume in almost all cases that substitutions $\theta$ satisfy the additional property that the set of time variables occurring in $\theta$ is exactly the set $\left\{t_{1}, \ldots, t_{n}\right\}$ of the first $n$ time variables. Substitutions will be notated as $\left[t_{1} / r_{1}, \ldots, t_{n} / r_{n}\right]$. With $\epsilon$ we denote the empty substitution. The mapping tvar gives for each substitution the time variables occurring in that substitution. We conclude this section with the definition of the metric on substitutions and the observation that this metric gives us a complete metric space.

Definition 2.5 The mapping $d_{S u b s t}:$ Subst $\times$ Subst $\rightarrow[0,1]$ is given by

$$
\begin{array}{lr}
d_{\text {Subst }}\left(\theta, \theta^{\prime}\right)=0 & \theta=\epsilon \wedge \theta^{\prime}=\epsilon \\
d_{\text {Subst }}\left(\theta, \theta^{\prime}\right)=\max \left\{d_{\mathbb{Z}}\left(\theta t, \theta^{\prime} t\right) \mid t \in \operatorname{tvar}(\theta)\right\} & \operatorname{tvar}(\theta)=\operatorname{tvar}\left(\theta^{\prime}\right) \\
d_{\text {Subst }}\left(\theta, \theta^{\prime}\right)=1 & \text { otherwise }
\end{array}
$$

\section{Operational semantics}

In this section we present an operational semantic model for our language. The operational semantics of a program describes the behaviour of an abstract machine running that program. The execution of a program on an abstract machine is characterised by sets of timed streams. Which timed actions and in which order the timed actions are performed by the abstract machine is described by a labelled transition system [18].

Before giving a labelled transition system, we first introduce the empty statement $E$. This empty statement is associated with termination. The class of statements Stat is extended to Stat $_{E}$.

Definition 3.1 The class $S t a t_{E}$, with typical element $\bar{s}$, is given by Stat $_{E}=\operatorname{Stat} \cup\{E\}$

Having extended the class of statements, we can also extend the notions of free time variables and closed statements. It will be convenient to allow expressions of the form $\bar{s} * \bar{s}^{\prime}$. This will reduce the number of rules of the labelled transition system. We define the following reasonable equivalences on these expressions.

Definition 3.2 For all $\bar{s} \in \operatorname{Stat}_{E}$ and $* \in\{i, U, \|\}$

$$
\bar{s} * E=\bar{s} \text { and } E * \bar{s}=\bar{s}
$$

Next we present a transition relation, which induces a labelled transition system as is described in, for example, [17].

Definition 3.3 The transition relation $\rightarrow$ is the smallest subset of CStat $\times T A \times \operatorname{Decl} \times$ CStat $_{E}$ satisfying

$$
\begin{aligned}
& (a, e)-(a, \mathcal{V}(e)) \rightarrow_{d} E \\
& \frac{g-\alpha \rightarrow_{d} \bar{s} \quad(x, g) \in d}{x-\alpha \rightarrow_{d} \bar{s}} \\
& \frac{s-\alpha \rightarrow_{d} \bar{s}}{s ; s^{\prime}-\alpha \rightarrow_{d} \bar{s} ; s^{\prime}}
\end{aligned}
$$




$$
\begin{aligned}
& \frac{s-\alpha \rightarrow{ }_{d} \bar{s}}{s \cup s^{\prime}-\alpha \rightarrow_{d} \bar{s}} \\
& s^{\prime} \cup s-\alpha \rightarrow{ }_{d} \bar{s} \\
& \frac{s-\alpha \rightarrow_{d} \bar{s}}{s\left\|s^{\prime}-\alpha \rightarrow_{d} \bar{s}\right\| s^{\prime}} \\
& s^{\prime}\left\|s-\alpha \rightarrow_{d} s^{\prime}\right\| \bar{s} \\
& \frac{s[t / r]-\alpha \rightarrow_{d} \bar{s} \quad r \in T}{\int_{t \in T} s-\alpha \rightarrow_{d} \bar{s}}
\end{aligned}
$$

Intuitively, a rule $s-\alpha \rightarrow_{d} \bar{s}$ tells us that the execution of statement $s$ consists of timed action $\alpha$ followed by the execution of statement $\vec{s}$. Consider the axiom for the statement $(a, e)$. The execution of $(a, e)$ consists of the execution of atomic action $a$ at $\mathcal{V}(e)$, the value of expression $e$, after its enabling followed by termination. Because $(a, e)$ is a closed statement, the evaluation of the expression $e$ delivers an element of $I R_{>}$. Since the evaluation of an expression delivers an element of $\mathbb{R}_{>}$, we can conclude that two successive atomic actions cannot be executed at the same time. The rule for a procedure call indicates body replacement. Parallel composition is modelled by arbitrary interleaving of the atomic actions of both statements. The rule for integration states that some arbitrary element $r$ from time set $T$ is passed to time variable $t$ in statement $s$. Using the above rules we can derive that $\int_{t \in[1.03,2.41]}(a, t)-(a, r) \rightarrow_{d} E$ for all $r \in[1.03,2.41]$.

Now we can define the operational semantics for closed statements $s$, related to a declaration $d$, such that all procedure variables occurring in statement $s$ or declaration $d$ are declared in declaration $d$.

Definition 3.4 The mapping $\mathcal{O}_{d}:$ CStat $\rightarrow \mathcal{P}(T S)$ is given by $\sigma \in \mathcal{O}_{d}(s)$ if and only if one of the following conditions is satisfied:

- $\exists n \in \mathbb{N}: \exists s_{1}, \ldots, s_{n} \in$ CStat $: \exists \alpha_{1}, \ldots, \alpha_{n+1} \in T A$ :

$$
\begin{aligned}
& s-\alpha_{1} \rightarrow_{d} s_{1}-\alpha_{2} \rightarrow_{d} \cdots-\alpha_{n} \rightarrow_{d} s_{n}-\alpha_{n+1} \rightarrow_{d} E \wedge \\
& \sigma=\left\langle\alpha_{1},\left\langle\alpha_{2}, \cdots<\alpha_{n}, \alpha_{n+1}>\cdots\right\rangle>\right. \\
& \text { • } \exists s_{1}, \ldots \in \text { CStat }: \exists \alpha_{1}, \ldots \in T A: \\
& s-\alpha_{1} \rightarrow_{d} s_{1}-\alpha_{2} \rightarrow_{d} \cdots \wedge \sigma=\left\langle\alpha_{1},\left\langle\alpha_{2}, \cdots\right\rangle\right\rangle
\end{aligned}
$$

For example, we have that $\mathcal{O}_{d}\left(\int_{t \in[1.03,2.41]}(a, t)\right)=\{(a, 1.03), \ldots,(a, 2.41)\}$. We conclude this section with the definition of the operational semantics for programs.

Definition 3.5 The mapping $\mathcal{O}: \operatorname{Prog} \rightarrow \mathcal{P}(T S)$ is given by

$$
\mathcal{O}((d, s))=\mathcal{O}_{d}(s)
$$

\section{Denotational semantics}

After having defined an operational semantics, we give a denotational semantics for the language. This denotational semantics is by definition compositional, i.e. the meaning of a program can be derived from the meaning of its constituent parts. Fixed points are used to deal with recursion. To obtain a compositional model we define for every syntactic operator $\square$ a corresponding semantic operator $\diamond$, such that we have, for example, 
$\mathcal{D}\left(\left(d, s_{1} \square s_{2}\right)\right)=\mathcal{D}\left(\left(d, s_{1}\right)\right) \diamond \mathcal{D}\left(\left(d, s_{2}\right)\right)$. To handle recursion we define the denotational model as a fixed point of a higher-order transformation. In this section we will use extensively Banach's fixed point theorem and Michael's theorem. Both theorems will be used for the construction of the semantic operators and the denotational model.

For the syntactic operator; we define a corresponding semantic operator, which will also be denoted by ;. First we define the semantic operator ; on timed streams. This operator will be defined as a fixed point of a higher-order transformation $\Psi_{i}$. This higherorder transformation is a contraction on a complete metric space. Using Banach's fixed point theorem, we can conclude that $\Psi_{\text {; }}$ has a unique fixed point, which we will denote by ;. Then we lift the semantic operator ; which has been defined on timed streams, such that we obtain a semantic operator defined on (non-empty compact) sets of timed streams. First we introduce the higher-order transformation $\Psi_{;}$.

Definition 4.1 The mapping $\Psi_{;}:\left(T S \times T S \rightarrow \mathcal{P}_{n c}(T S)\right) \rightarrow\left(T S \times T S \rightarrow \mathcal{P}_{n c}(T S)\right)$ is given by

$$
\begin{aligned}
& \left.\Psi_{i}(F)(\alpha, \tau)=\{<\alpha, \tau\rangle\right\} \\
& \Psi_{;}(F)(\langle\alpha, \sigma\rangle, \tau)=\{<\alpha, \rho>\mid \rho \in F(\sigma, \tau)\}
\end{aligned}
$$

It is obvious that the mapping $\Psi_{i}$ is well-defined. Furthermore, it can be shown that this mapping is a contraction. Because $\Psi_{i}$ is a contraction on a complete metric space, we can deduce using Banach's fixed point theorem that $\Psi_{;}$has a unique fixed point, which will be denoted by ;.

Corollary 4.2 The operator ; $T S \times T S \rightarrow \mathcal{P}_{n c}(T S)$ given by

$$
\begin{aligned}
& \alpha ; \tau=\{\langle\alpha, \tau\rangle\} \\
& \langle\alpha, \sigma\rangle_{;} \tau=\{\langle\alpha, \rho\rangle \mid \rho \in \sigma ; \tau\}
\end{aligned}
$$

is well-defined.

Before we can lift the semantic operator ; we have to show that this operator is continuous. We can show that the operator; is non-distance increasing in its first argument and contracting with factor $\frac{1}{2}$ in its second argument using fixed point induction and Banach's fixed point theorem without using the definition of the metric $d_{\mathbb{R}_{\geq}}$.

Property 4.3 For all $\sigma, \sigma^{\prime}, \tau, \tau^{\prime} \in T S$ and $\varepsilon \in I R \geq$ $d_{T S}\left(\sigma, \sigma^{\prime}\right) \leq \varepsilon \wedge d_{T S}\left(\tau, \tau^{\prime}\right) \leq 2 \varepsilon \Rightarrow d_{\mathcal{P}_{n c}(T S)}\left(\sigma ; \tau, \sigma^{\prime} ; \tau^{\prime}\right) \leq \varepsilon$

The operator; is lifted to sets of timed streams in the following definition.

Definition 4.4 The operator;: $\mathcal{P}_{n c}(T S) \times \mathcal{P}_{n c}(T S) \rightarrow \mathcal{P}_{n c}(T S)$ is given by $S ; T=\bigcup\{\sigma ; \tau \mid \sigma \in S \wedge \tau \in T\}$

We can show the well-definedness of the lifted operator; using Michael's theorem and the fact that the operator; is continuous. Also for the lifted operator in we can show that it is non-distance increasing in its first argument and contracting with factor $\frac{1}{2}$ in its second argument. This property will be used to prove the well-definedness of the denotational semantics.

Property 4.5 For all $S, S^{\prime}, T, T^{\prime} \in \mathcal{P}_{n c}(T S)$ and $\varepsilon \in I R_{\geq}$

$$
d_{\mathcal{P}_{n e}(T S)}\left(S, S^{\prime}\right) \leq \varepsilon \wedge d_{\mathcal{P}_{n \in}(T S)}\left(T, T^{\prime}\right) \leq 2 \varepsilon \Rightarrow d_{\mathcal{P}_{n e}(T S)}\left(S ; T, S^{\prime} ; T^{\prime}\right) \leq \varepsilon
$$


Next we define two semantic operators which correspond to the syntactic notions of non-deterministic choice and integration.

Definition 4.6 The operator $U: \mathcal{P}_{n c}(T S) \times \mathcal{P}_{n c}(T S) \rightarrow \mathcal{P}_{n c}(T S)$ is defined as the set-theoretic union and the operator $\bigcup: \mathcal{P}_{n c}\left(\mathcal{P}_{n c}(T S)\right) \rightarrow \mathcal{P}_{n c}(T S)$ is defined as the generalised set-theoretic union.

From Michael's theorem we can conclude that the semantic operator $\bigcup$ is well-defined. The semantic counterpart of the syntactic operator $\|$ is also defined as the unique fixed point of a higher-order transformation. This higher-order transformation $\Psi_{\|}$is defined by means of the higher-order transformation $\Psi_{i}$.

Definition 4.7 The mapping $\Psi_{\|}:\left(T S \times T S \rightarrow \mathcal{P}_{n c}(T S)\right) \rightarrow\left(T S \times T S \rightarrow \mathcal{P}_{n c}(T S)\right)$ is given by

$$
\Psi_{\|}(F)(\sigma, \tau)=\Psi_{i}(F)(\sigma, \tau) \cup \Psi_{i}(F)(\tau, \sigma)
$$

The well-definedness of the mapping $\Psi_{\|}$follows from the well-definedness of $\Psi_{i}$. From the fact that $\Psi_{i}$ is a contraction we can deduce that $\Psi_{\|}$is also a contraction. We denote the unique fixed point of the higher-order transformation $\Psi_{\|}$by $\|$. We can define $\|$by means of a so-called left-merge $U$, which expresses a merge where the first element is taken from the left argument. We have that

$$
\sigma \| \tau=\Psi_{\|}(\|)(\sigma, \tau)=\Psi_{;}(\|)(\sigma, \tau) \cup \Psi_{i}(\|)(\tau, \sigma)
$$

Furthermore

and

$$
\left.\Psi_{i}(\|)(\alpha, \tau)=\{<\alpha, \tau\rangle\right\}
$$

$$
\Psi_{i}(\|)(\langle\alpha, \sigma\rangle, \tau)=\{\langle\alpha, \rho\rangle \mid \rho \in \sigma \| \tau\}
$$

Thus we can characterise the operators $\|$ and $\mathbb{L}$ as follows.

Corollary 4.8 The operator $\|: T S \times T S \rightarrow \mathcal{P}_{n c}(T S)$ given by

$$
\sigma\|\tau=\sigma\| \tau \cup \tau \| \sigma
$$

and the operator $\mathbb{U}: T S \times T S \rightarrow \mathcal{P}_{n e}(T S)$ given by

$$
\begin{aligned}
& \alpha \| \tau=\{\langle\alpha, \tau\rangle\} \\
& \langle\alpha, \sigma\rangle \| \tau=\{\langle\alpha, \rho>| \rho \in \sigma \| \tau\}
\end{aligned}
$$

are well-defined.

Because both semantic operators are continuous, we can lift these operators.

Definition 4.9 The operator $\|: \mathcal{P}_{n c}(T S) \times \mathcal{P}_{n c}(T S) \rightarrow \mathcal{P}_{n c}(T S)$ is given by $S \| T=S\lfloor T \cup T \| S$

and the operator $\amalg: \mathcal{P}_{n c}(T S) \times \mathcal{P}_{n c}(T S) \rightarrow \mathcal{P}_{n c}(T S)$ is given by $S \| T=\bigcup\{\sigma \| \tau \mid \sigma \in S \wedge \tau \in T\}$

Having defined the semantic operators, we can define the denotational semantics for statements related to a declaration, such that all procedure variables occurring in the statement or the declaration are declared in the declaration. To obtain a compositional model we introduce substitutions, which record the choices made for the time variables with respect to integration. For example, to derive the meaning of the statement $\int_{t \in[1.03,2.41]}(a, t)$ from the meaning of the statement $(a, t)$, we record the choice made for the time variable $t$ in the substitutions $[t / r]$ for $r \in[1.03,2.41]$. 
We define the denotational semantics $\mathcal{D}_{d}:$ Stat $\rightarrow S u b s t \rightarrow_{p} \mathcal{P}_{n c}(T S)$ as a partial function such that $\mathcal{D}_{d}(s)(\theta)$ is defined whenever $\operatorname{tvar}(s) \subseteq \operatorname{tvar}(\theta)$. The substitution $\theta$ should bind all free variables of statement $s$. We define the denotational semantics $\mathcal{D}_{d}$ as the fixed point of a higher-order transformation $\Psi_{\mathcal{D}}$. We have to impose a restriction on this mapping $\Psi_{\mathcal{D}}$ in order to obtain a well-defined mapping. We restrict $\Psi_{\mathcal{D}}$ to a mapping from [Stat $\rightarrow$ Subst $\left.\rightarrow_{p} \mathcal{P}_{n c}(T S)\right]$, the collection of continuous mappings Stat $\rightarrow$ Subst $\rightarrow_{p} \mathcal{P}_{n c}(T S)$, to $\left[\right.$ Stat $\rightarrow$ Subst $\left.\rightarrow_{p} \mathcal{P}_{n c}(T S)\right]$

Definition 4.10 The mapping $\Psi_{\mathcal{D}}:\left[\right.$ Stat $\rightarrow$ Subst $\left.\rightarrow_{p} \mathcal{P}_{n c}(T S)\right] \rightarrow\left[\right.$ Stat $\rightarrow$ Subst $\rightarrow_{p}$ $\left.\mathcal{P}_{n a}(T S)\right]$ is given by

$$
\begin{array}{ll}
\Psi_{\mathcal{D}}(F)((a, e))(\theta)=\{(a, \mathcal{V}(e \theta))\} & \\
\Psi_{\mathcal{D}}(F)(x)(\theta)=\Psi_{\mathcal{D}}(F)(g)(\theta) & (x, g) \in d \\
\Psi_{\mathcal{D}}(F)\left(s_{1} ; s_{2}\right)(\theta)=\Psi_{\mathcal{D}}(F)\left(s_{1}\right)(\theta) ; F\left(s_{2}\right)(\theta) & \\
\Psi_{\mathcal{D}}(F)\left(s_{1} * s_{2}\right)(\theta)=\Psi_{\mathcal{D}}(F)\left(s_{1}\right)(\theta) * \Psi_{\mathcal{D}}(F)\left(s_{2}\right)(\theta) & * \in\{U, \|\} \\
\Psi_{\mathcal{D}}(F)\left(\int_{t \in T} s\right)(\theta)=\bigcup\left\{\Psi_{\mathcal{D}}(F)(s)(\theta[t / r]) \mid r \in T\right\} &
\end{array}
$$

Because expressions can be associated with continuous functions, time sets are non-empty compact sets and the semantic operators are continuous, we can show that the higherorder transformation $\Psi_{\mathcal{D}}$ is well-defined using Michael's theorem. We can show that $\Psi_{\mathcal{D}}$ is a contraction. The contractiveness of $\Psi_{\mathcal{D}}$ follows from the contraction properties of the semantic operators. Because $\Psi_{D}$ is a contraction on a complete metric space, this mapping has a unique fixed point, which we will denote by $\mathcal{D}_{d}$. We can characterise $\mathcal{D}_{d}$ as follows.

Corollary 4.11 The mapping $\mathcal{D}_{d}:$ Stat $\rightarrow S u b s t \rightarrow_{p} \mathcal{P}_{n c}(T S)$ is given by

$$
\begin{aligned}
& \mathcal{D}_{d}((a, e))(\theta)=\{(a, \mathcal{V}(e \theta))\} \\
& \mathcal{D}_{d}(x)(\theta)=\mathcal{D}_{d}(g)(\theta) \\
& \mathcal{D}_{d}\left(s_{1} * s_{2}\right)(\theta)=\mathcal{D}_{d}\left(s_{1}\right)(\theta) * \mathcal{D}_{d}\left(s_{2}\right)(\theta) \\
& \mathcal{D}_{d}\left(\int_{t \in T} s\right)(\theta)=\bigcup\left\{\mathcal{D}_{d}(s)(\theta[t / r]) \mid r \in T\right\}
\end{aligned}
$$

We have, for example,

$$
\begin{aligned}
\mathcal{D}_{d}\left(\int_{t \in[1.03,2.41]}(a, t)\right)(\epsilon) & =\bigcup\left\{\mathcal{D}_{d}((a, t))([t / r]) \mid r \in[1.03,2.41]\right\} \\
& =\bigcup\{\{(a, t[t / r])\} \mid r \in[1.03,2.41]\} \\
& =\{(a, r) \mid r \in[1.03,2.41]\}
\end{aligned}
$$

The denotational semantics for programs is defined as follows.

Definition 4.12 The mapping $\mathcal{D}: \operatorname{Prog} \rightarrow \mathcal{P}_{n c}(T S)$ is given by

$$
\mathcal{D}((d, s))=\mathcal{D}_{d}(s)(\epsilon)
$$

\section{Equivalence proof}

Having defined both an operational and a denotational semantics for our language the question arises whether the denotational model is correct with respect to the computational intuition captured by the operational model. In this section we will show that we can relate the operational model $\mathcal{O}$ and the denotational model $\mathcal{D}$. We will show that these models are equivalent. To show this we will use a general method for comparing different semantic models, which is described in [23]: if two models are both a fixed point 
of a higher-order transformation and this higher-order transformation is a contraction on a complete metric space, we can conclude that those models are equivalent.

We will introduce an intermediate operational semantic model $\mathcal{O}_{d}^{*}$ and relate this model to the operational model $\mathcal{O}_{d}$. Furthermore, we will define an intermediate denotational semantic model $\mathcal{D}_{d}^{*}$ and relate this model to the denotational model $\mathcal{D}_{d}$. Finally, we will introduce a higher-order transformation $\Psi_{\mathcal{O}^{*} \mathcal{D}^{*}}$, which is a contraction on a complete metric space. We can show that $\Psi_{\mathcal{O}^{*} \mathcal{D}^{*}}\left(\mathcal{O}_{d}^{*}\right)=\mathcal{O}_{d}^{*}$ and $\Psi_{\mathcal{O}^{*} \mathcal{D}^{*}}\left(\mathcal{D}_{d}^{*}\right)=\mathcal{D}_{d}^{*}$. From this we can conclude that $\mathcal{O}_{d}^{*}$ and $\mathcal{D}_{d}^{*}$ are equivalent. These relations will enable us to show the equivalence of $\mathcal{O}$ and $\mathcal{D}$.

First an intermediate operational model $\mathcal{O}_{d}^{*}$, which is associated with a labelled transition system, is introduced. In this operational model statements and substitutions, which record choices made for time variables with respect to integration, are separated. Therefore we introduce the class of configurations.

Definition 5.1 The class Conf of configurations, with typical element $C$, is given by Conf $=\{[s, \theta] \in S t a t \times S u b s t \mid \operatorname{tvar}(s) \subseteq \operatorname{tvar}(\theta)\}$

and the class $\operatorname{Conf}_{E}$ of configurations, with typical element $\bar{C}$, is given by

$\operatorname{Conf}_{B}=\left\{[\bar{s}, \theta] \in S \operatorname{tat}_{E} \times S u b s t \mid \operatorname{tvar}(\bar{s}) \subseteq \operatorname{tvar}(\theta)\right\}$

The transition relation, which induces the labelled transition system describing the intermediate operational semantics, is presented in the following definition.

Definition 5.2 The transition relation $\longrightarrow$ is the smallest subset of Conf $\times T A \times$ Decl $\times$ $\operatorname{Conf}_{E}$ satisfying

$$
\begin{aligned}
& {[(a, e), \theta]-(a, \mathcal{V}(e \theta)) \rightarrow_{d}[E, \theta]} \\
& \frac{[g, \theta]-\alpha \rightarrow_{d} \ddot{C} \quad(x, g) \in d}{[x, \theta]-\alpha \rightarrow_{d} \bar{C}} \\
& \frac{[s, \theta]-\alpha \rightarrow_{d}\left[\bar{s}, \theta^{\prime}\right]}{\left[s ; s^{\prime}, \theta\right]-\alpha \rightarrow_{d}\left[\bar{s}_{;} s^{\prime}, \theta^{\prime}\right]} \\
& \frac{[s, \theta]-\alpha \rightarrow{ }_{d} \bar{C}}{\left[s \cup s^{\prime}, \theta\right]-\alpha \rightarrow_{d} \bar{C}} \\
& {\left[s^{\prime} \cup s, \theta\right]-\alpha \rightarrow_{d} \bar{C}} \\
& \frac{[s, \theta]-\alpha \rightarrow_{d}\left[\bar{s}, \theta^{\prime}\right]}{\left[s \| s^{\prime}, \theta\right]-\alpha \rightarrow_{d}\left[\bar{s} \| s^{\prime}, \theta^{\prime}\right]} \\
& {\left[s^{\prime} \| s, \theta\right]-\alpha \rightarrow_{d}\left[s^{\prime} \| \bar{s}, \theta^{\prime}\right]} \\
& {\left[s\left[t / t_{n+1}\right], \theta\left[t_{n+1} / r\right]\right]-\alpha \rightarrow_{d} \bar{C} \quad \operatorname{tvar}(\theta)=\left\{t_{1}, \ldots, t_{n}\right\}} \\
& \hline \quad\left[\int_{t \in T} s, \theta\right]-\alpha \rightarrow_{d} \bar{C}
\end{aligned}
$$

The intermediate operational model $\mathcal{O}_{d}^{*}$ is defined along the lines of the definition of the operational model $\mathcal{O}_{d}$.

Definition 5.3 The mapping $\mathcal{O}_{d}^{*}:\left[\operatorname{Conf} \rightarrow \mathcal{P}_{n c}(T S)\right]$ is given by $\sigma \in \mathcal{O}_{d}^{*}(C)$ if and only if one of the following conditions is satisfied: 
- $\exists n \in \mathbb{I N}: \exists C_{1}, \ldots, C_{n} \in$ Conf $: \exists \alpha_{1}, \ldots, \alpha_{n+1} \in T A: \exists \theta \in$ Subst :

$C-\alpha_{1} \rightarrow_{d} C_{1}-\alpha_{2} \rightarrow_{d} \cdots-\alpha_{n} \rightarrow_{d} C_{n}-\alpha_{n+1} \rightarrow_{d}[E, \theta] \wedge$

$\sigma=\left\langle\alpha_{1},\left\langle\alpha_{2}, \cdots<\alpha_{n}, \alpha_{n+1}\right\rangle \cdots\right\rangle>$

- $\exists C_{1}, \ldots \in$ Conf $: \exists \alpha_{1}, \ldots \in T A$ :

$C-\alpha_{1} \rightarrow_{d} C_{1}-\alpha_{2} \rightarrow_{d} \cdots \wedge \sigma=\left\langle\alpha_{1},\left\langle\alpha_{2}, \cdots\right\rangle\right\rangle$

The well-definedness of the intermediate operational semantics follows from some properties of the labelled transition system. Next we relate the operational models $\mathcal{O}_{d}$ and $\mathcal{O}_{d}^{*}$ via their labelled transition systems. From the following property we can deduce that each step according to the labelled transition system describing $\mathcal{O}_{d}$ can be mimicked by a step according to the labelled transition system describing $\mathcal{O}_{d}^{*}$ and vice versa.

Property 5.4 For all $[s, \theta] \in$ Conf, $\bar{s} \in$ Stat $_{E}$ and $\alpha \in T A$

$s \theta-\alpha \rightarrow_{d} \bar{s} \Leftrightarrow \exists\left[\bar{s}^{\prime}, \theta^{\prime}\right] \in \operatorname{Conf}_{E}:[s, \theta]-\alpha \rightarrow_{d}\left[\bar{s}^{\prime}, \theta^{\prime}\right] \wedge \bar{s}=\bar{s}^{\prime} \theta^{\prime}$

Having related the labelled transition systems, which describe the operational models $\mathcal{O}_{d}$ and $\mathcal{O}_{d}^{*}$, we can relate these models.

Lemma 5.5 For all $[s, \theta] \in$ Conf

$$
\mathcal{O}_{d}^{*}([s, \theta])=\mathcal{O}_{d}(s \theta)
$$

Now we introduce an intermediate denotational model $\mathcal{D}_{d}^{*}$. This denotational model is defined as the fixed point of a higher-order transformation $\Psi_{\mathcal{D}^{*}}$.

Definition 5.6 The mapping $\Psi_{\mathcal{D}^{\bullet}}:\left[\right.$ Conf $\left.\rightarrow \mathcal{P}_{n c}(T S)\right] \rightarrow\left[\right.$ Conf $\left.\rightarrow \mathcal{P}_{n c}(T S)\right]$ is given by

$$
\begin{array}{lr}
\Psi_{\mathcal{D}^{*}}(F)([(a, e), \theta])=\{(a, \mathcal{V}(e \theta))\} & \\
\Psi_{\mathcal{D}^{*}}(F)([x, \theta])=\Psi_{\mathcal{D}^{*}}(F)([g, \theta]) & (x, g) \in d \\
\Psi_{\mathcal{D}^{*}}(F)\left(\left[s_{1} ; s_{2}, \theta\right]\right)=\Psi_{\mathcal{D}^{*}}(F)\left(\left[s_{1}, \theta\right]\right) ; F\left(\left[s_{2}, \theta\right]\right) & \\
\Psi_{\mathcal{D}^{*}}(F)\left(\left[s_{1} * s_{2}, \theta\right]\right)=\Psi_{\mathcal{D}^{*}}(F)\left(\left[s_{1}, \theta\right]\right) * \Psi_{\mathcal{D}^{*}}(F)\left(\left[s_{2}, \theta\right]\right) & * \in\{U, \|\} \\
\Psi_{\mathcal{D}^{*}}(F)\left(\left[\int_{t \in T} s, \theta\right]\right)=\bigcup\left\{\Psi_{\mathcal{D}^{*}}(F)\left(\left[s\left[t / t_{n+1}\right], \theta\left[t_{n+1} / r\right]\right]\right) \mid r \in T\right\} & \\
& \operatorname{tvar}(\theta)=\left\{t_{1}, \ldots, t_{n}\right\}
\end{array}
$$

Using Michael's theorem we can show that the mapping $\Psi_{\mathcal{D}^{*}}$ is well-defined, because expressions are continuous functions, time sets are non-empty compact sets and the semantic operators are continuous. Next we can show that $\Psi_{\mathcal{D}^{*}}$ is a contraction, which follows from the contraction properties of the semantic operators. The mapping $\Psi_{\mathcal{D}^{*}}$ has a unique fixed point, because this mapping is a contraction on a complete metric space. We will denote this fixed point by $\mathcal{D}_{d}^{*}$.

Corollary 5.7 The mapping $\mathcal{D}_{d}^{*}:\left[\right.$ Conf $\left.\rightarrow \mathcal{P}_{n c}(T S)\right]$ given by

$$
\begin{array}{lr}
\mathcal{D}_{d}^{*}([(a, e), \theta])=\{(a, \mathcal{V}(e \theta))\} & \\
\mathcal{D}_{d}^{*}([x, \theta])=\mathcal{D}_{d}^{*}([g, \theta]) & (x, g) \in d \\
\mathcal{D}_{d}^{*}\left(\left[s_{1} * s_{2}, \theta\right]\right)=\mathcal{D}_{d}^{*}\left(\left[s_{1}, \theta\right]\right) * \mathcal{D}_{d}^{*}\left(\left[s_{2}, \theta\right]\right) & * \in\{i, \cup, \|\} \\
\mathcal{D}_{d}^{*}\left(\left[\int_{t \in T} s, \theta\right]\right)=\bigcup\left\{\mathcal{D}_{d}^{*}\left(\left[s\left[t / t_{n+1}\right], \theta\left[t_{n+1} / r\right]\right]\right) \mid r \in T\right\} & \operatorname{tvar}(\theta)=\left\{t_{1}, \ldots, t_{n}\right\}
\end{array}
$$

is well-defined.

The denotational models $\mathcal{D}_{d}^{*}$ and $\mathcal{D}_{d}$ can be related by showing that $\mathcal{D}_{d}^{*}$ is a fixed point of the higher-order transformation $\Psi_{\mathcal{D}}$. 
Lemma 5.8 For all $[s, \theta] \in$ Conf

$$
\mathcal{D}_{d}^{*}([s, \theta])=\mathcal{D}_{d}(s)(\theta)
$$

We can relate the intermediate models $\mathcal{O}_{d}^{*}$ and $\mathcal{D}_{d}^{*}$ by showing that they are both a fixed point of the higher-order transformation $\Psi_{\mathcal{O}^{*} \mathcal{D}^{*}}$. This mapping $\Psi_{\mathcal{O}^{*} \mathcal{D}^{*}}$ is related to a declaration similar to the denotational models.

Definition 5.9 The mapping $\Psi_{\mathcal{O}^{*} \mathcal{D}^{*}}:\left[\right.$ Conf $\left.\rightarrow \mathcal{P}_{n c}(T S)\right] \rightarrow\left[\right.$ Conf $\left.\rightarrow \mathcal{P}_{n c}(T S)\right]$ is given by

$$
\Psi_{\mathcal{O} \cdot \mathcal{D} \cdot}(F)(C)=\left\{\langle\alpha, \rho\rangle \mid C-\alpha \rightarrow_{d} C^{\prime} \wedge \rho \in F\left(C^{\prime}\right)\right\} \cup\left\{\alpha \mid C-\alpha \rightarrow_{d}\left[E, \theta^{\prime}\right]\right\}
$$

The well-definedness of the higher-order transformation $\Psi_{\mathcal{O}} \cdot \mathcal{D}^{*}$ follows from some properties of the labelled transition system. Because $\Psi_{\mathcal{O}^{*} \mathcal{D}^{*}}$ is a contraction on a complete metric space, we can conclude that this mapping has a unique fixed point. We can show that the intermediate operational model $\mathcal{O}_{d}^{*}$ is a fixed point of $\Psi_{\mathcal{O}^{*} D^{*}}$.

Lemma $5.10 \Psi_{\mathcal{O} \cdot D^{*}}\left(\mathcal{O}_{d}^{*}\right)=\mathcal{O}_{d}^{*}$

Also the intermediate denotational model $\mathcal{D}_{d}^{*}$ is a fixed point of $\Psi_{\mathcal{O}^{*} \mathcal{D}^{*}}$.

Lemma $5.11 \Psi_{\mathcal{O} \cdot \mathcal{D} \cdot}\left(\mathcal{D}_{d}^{*}\right)=\mathcal{D}_{d}^{*}$

Because $\mathcal{O}_{d}^{*}$ and $\mathcal{D}_{d}^{*}$ are both fixed points of the higher-order transformation $\Psi_{\mathcal{O}^{*} \mathcal{D}^{*}}$, which is a contraction on a complete metric space, we have that $\mathcal{O}_{d}^{*}$ and $\mathcal{D}_{d}^{*}$ are equivalent due to Banach's fixed point theorem.

Corollary $5.12 \mathcal{O}_{d}^{*}=\mathcal{D}_{d}^{*}$

We conclude this section by collecting all the relations between the various models, which are stated in lemma 5.5, lemma 5.8 and corollary 5.12 , into an equivalence result.

Theorem 5.13 $\mathcal{O}=\mathcal{D}$

\section{Conclusions}

An operational and a denotational semantic model have been presented for a real-time programming language incorporating the concept of integration. As we have seen, a restricted form of unbounded non-determinism can be specified by means of integration. Because the semantic operators and the semantic models have been defined using higherorder transformations, we were able to describe infinite behaviour. The operational and denotational semantics have been proved equivalent. Banach's fixed point theorem and Michael's theorem have been used fruitfully to define and to compare those models.

We expect it to be possible to relate the denotational semantics defined in $[26,27]$ to a denotational model based on the denotational model presented in this paper following the lines of [6]. We have the strong feeling that it is possible to extend the language with communication and global non-determinism [5] and to define a branching time model [3] for this language. Enriching the language with delays $[24,27]$ and a parameter mechanism provided to procedure variables causes no serious problems. However, extending the language with priorities $[13,29]$ and enforced deadlines for atomic actions may cause discontinuity of semantic operators. 


\section{Acknowledgements}

I would like to thank the members of the Amsterdam Concurrency Group for comments on previous work. In particular, I thank Erik de Vink for his comments and suggestions during the evolvement of this paper.

\section{References}

[1] K.R. Apt and G.D. Plotkin. Countable Non-Determinism and Random Assignment. Journal of the ACM 33 (1986), 724-767.

[2] J.C.M. Baeten and J.A. Bergstra. Real-Time Process Algebra. Report P8916, Programming Research Group, University of Amsterdam, Amsterdam (1989).

[3] J.W. de Bakker, J.A. Bergstra, J.W. Klop and J.-J.Ch. Meyer. Linear Time and Branching Time Semantics for Recursion with Merge. Theoretical Computer Science 34 (1984), 135156.

[4] J.W. de Bakker and J.N. Kok. Comparative Metric Semantics for Concurrent Prolog. Theoretical Computer Science 75 (1990), 15-43.

[5] J.W. de Bakker, J.N. Kok, J.-J.Ch. Meyer, E.-R. Olderog and J.I. Zucker. Contrasting Themes in the Semantics of Imperative Concurrency. Current Trends in Concurrency: Overviews and Tutorials (J.W. de Bakker, W.P. de Roever and G. Rozenberg, eds.), Lecture Notes in Computer Science 224, Springer (1986), 51-121.

[6] J.W. de Bakker, J.-J.Ch. Meyer and E.-R. Olderog. Infinite Streams and Finite Observations in the Semantics of Uniform Concurrency. Theoretical Computer Science 49 (1987), $87-112$.

[7] J.W. de Bakker and J.-J.Ch. Meyer. Metric Semantics for Concurrency. BIT 28 (1988), 504-529.

[8] J.W. de Bakker and E.P. de Vink. CCS for $O O$ and LP. This volume.

[9] J.W. de Bakker and J.I. Zucker. Processes and the Denotational Semantics of Concurrency. Information and Control 54 (1982), 70-120.

[10] G. Berry and L. Cosserat. The ESTEREL Synchronous Programming Language and its Semantics. Proceedings CMU Seminar on Concurrency (S.D. Brookes, A.W. Roscoe and G. Winksel, eds.), Lecture Notes in Computer Science 197, Springer (1985), 389-448.

[11] F. van Breugel. Semantic Models for a Language with Timed Atomic Actions. Report IR218, Vrije Universiteit, Amsterdam (1990).

[12] F. van Breugel. Comparative Semantics for a Real-Time Programming Language with Integration. Report IR-239, Vrije Universiteit, Amsterdam (1991).

[13] R. Cleaveland and M. Hennessy. Priorities in Process Algebra. Information and Computation $87(1990), 58-77$.

[14] J.W. Davies and S.A. Schneider. An Introduction to Timed CSP. Technical Monograph PRG-75, Oxford University Computing Laboratory, Oxford (1989). 
[15] A. Goswami and M. Joseph. Semantics of Real-Time Distributed Programs. Proceedings International Conference on Concurrency (F.H. Vogt, ed.), Lecture Notes in Computer Science 335, Springer (1988), 292-306.

[16] J.F. Groote. Specification and Verification of Real-Time Systems in ACP. Proceedings 10th International IFIP WG 6.1, Symposium on Protocol Specification, Testing and Verification, Ottawa (1990), 259-270.

[17] J.F. Groote and F.W. Vaandrager. Structured Operational Semantics and Bisimulation as a Congruence. Proceedings 16th International Colloquium on Automata, Languages and Programming (G. Ausiello, M. Dezani-Ciancaglini and S. Ronchi Della Rocca, eds.), Lecture Notes in Computer Science 372, Springer (1989), 423-438.

[18] M. Hennessy and G.D. Plotkin. Full Abstraction for a Simple Parallel Programming Language. Proceedings 8th Mathematical Foundations of Computer Science (J. Bečvar̆, ed.), Lecture Notes in Computer Science 74, Springer (1979), 108-120.

[19] M. Hennessy and T. Regan. A Temporal Process Algebra. Report 2-90, University of Sussex, Brighton (1990).

[20] W.H. Hesselink. Interpretations of Recursion under Unbounded Non-Determinacy. Theoretical Computer Science 59 (1988), 211-234.

[21] C.A.R. Hoare. Communicating Sequential Processes. Prentice-Hall International (1985).

[22] F. Jahanian and A. Mok. Safety Analysis of Timing Properties in Real-Time Systems. IEEE Transactions on Software Engineering 12 (1986), 890-904.

[23] J.N. Kok and J.J.M.M. Rutten. Contractions in Comparing Concurrency Semantics. Theoretical Computer Science 76 (1990), 179-222.

[24] R. Koymans, R.K. Shyamasundar, W.P. de Roever, R. Gerth and S. Arun-Kumar. Compositional Semantics for Real-Time Distributed Computing. Information and Computation 79 (1988), 210-256.

[25] M. Nivat. Infinite Words, Infinite Trees, Infinite Computations. Foundations of Computer Science (J.W. de Bakker and J. van Leeuwen, eds.), Mathematical Centre Tracts 109, Mathematical Centre, Amsterdam (1979), 3-52.

[26] G.M. Reed. A Hierarchy of Domains for Real-Time Distributed Computing. To appear in Proceedings Mathematical Foundations of Programming Languages and Semantics, Lecture Notes in Computer Science, Springer (1991).

[27] G.M. Reed and A.W. Roscoe. A Timed Model for Communicating Sequential Processes. Theoretical Computer Science 58 (1988), 249-261.

[28] J.J.M.M. Rutten. Semantic Correctness for a Parallel Object-Oriented Language. SIAM Journal of Computation 19 (1990), 341-383.

[29] S.A. Smolka and B. Steffen. Priority as Extremal Probability. Proceedings Conference on Theories of Concurrency: Unification and Extension (J.C.M. Baeten and J.W. Klop, eds.), Lecture Notes in Computer Science 458 (1990), 456-466.

[30] J.A. Stankovic. A Serious Problem for Next-Generation Systems. Computer 21 (1988), 10-19.

[31] N. Wirth. Towards a Discipline of Real-Time Programming. Communications of the ACM 20 (1977), 577-583. 